\title{
The effect of protein-protein interactions on the activity of APE1 SNP forms
}

\author{
Olga A. Kladova \\ Laboratory of biopolymer modification \\ Institute of chemical biology and \\ fundamental medicine \\ Novosibirsk, Russia \\ kladova@niboch.nsc.ru
}

\author{
Irina V. Alekseeva \\ Laboratory of biopolymer modification \\ Institute of chemical biology and \\ fundamental medicine \\ Novosibirsk, Russia \\ Irina.Alekseeva@niboch.nsc.ru \\ Nikita A. Kuznetsov \\ Laboratory of biopolymer modification \\ Institute of chemical biology and \\ fundamental medicine \\ Novosibirsk, Russia \\ Nikita.Kuznetsov@niboch.nsc.ru
}

\author{
Olga S. Fedorova \\ Laboratory of biopolymer modification \\ Institute of chemical biology and \\ fundamental medicine \\ Novosibirsk, Russia \\ fedorova@niboch.nsc.ru
}

\begin{abstract}
Human apurinic/apyrimidinic endonuclease 1 (APE1) is known to be an important participant in base excision repair (BER) and activator of several transcription factors. It was previously shown that APE1 can stimulate the activity of some DNA glycosylases, which indicates possible protein-protein interactions between APE1 and other BER enzymes. In this study, we tested several natural singlenucleotide polymorphic variants of APE1 (R221C, N222H, R237A, G241R, M270T, R274Q, P311S) on its ability to influence on protein-protein interactions with different human DNA repair enzymes such as OGG1, UNG, AAG, XRCC1, PCNA, and Pol $\beta$. Obtained data indicate differences in the BER enzyme effect on some APE1 variants supporting the important role of protein-protein interactions in the coordination of repair enzymes.
\end{abstract}

Keywords - base excision repair, protein-protein interactions, fluorescence

\section{Introduction}

One of the major pathways to remove DNA lesions is the base excision repair (BER). BER pathway is a multi-step process, and can be reconstituted with a limited number of proteins such as damage-specific DNA glycosylases, AP endonuclease, DNA polymerase, DNA ligase and additional coordination proteins XRCC1 and PCNA. Coordination of the enzymatic activities of DNA glycosylases and AP endonuclease is essential to ensure a complete repair of the damaged bases. A "passing-the-baton" model proposed for BER is consistent with the findings that DNA glycosylases coordinate with other proteins to process the damaged DNA substrate. Indeed, numerous studies have revealed that APE1 promotes dissociation of the DNA glycosylaseproduct complex, and this situation in turn increases multiple turnover rates of some DNA glycosylases. Two mechanisms were proposed to explain the stimulation of DNA glycosylases by APE1: a passive mechanism, when an AP endonuclease cleaves a free AP-site to prevent its rebinding with the DNA glycosylase during reaction and an active mechanism that consists of direct displacement of DNA glycosylase from the AP site either via specific protein-protein interactions or via distortion of the duplex DNA structure to disrupt the DNA glycosylase-AP site DNA complex. Nevertheless, the detailed molecular mechanism of DNA glycosylase stimulation by APE1 remains unknown.
In this study, we focused on the issue of damaged DNA transfer from the complex with various human DNA glycosylases (OGG1, UNG, AAG), DNA polymerase $\beta$, XRCC1 or PCNA to AP endonuclease APE1. We tested several single-nucleotide polymorphic (SNP) variants of APE1 (R221C, N222H, R237A, G241R, M270T, R274Q, $\mathrm{P} 311 \mathrm{~S})$ on its ability to influence on protein-protein interactions with different human DNA repair enzymes. SNP variants have substitutions of amino acid residues on the surface of the enzyme globule and in the DNA-binding site, thereby affecting protein-protein interactions or the catalytic reaction, respectively (Table 1).

\section{Materials and Methods}

The real time cleavage of the DNA duplex containing stable AP-site analog (F-site) was detected by stopped-flow method with fluorescence detection. A FAM/BHQ1-labeled substrate 5'-FAM-GCTCAFGTACAGAGCTG-3'/5'CAGCTCTGTACGTGAGC-BHQ1-3' was used to FRET analysis of the DNA cleavage reaction. The F-site cleavage activity of WT APE1 and SNP forms was estimated by calculating observed catalytic rate constant $k_{o b s}$. The activity of SNP variants in the presence of other BER enzymes was also studied by direct PAGE analysis of the product accumulation. Typical experiment was conducted at $37^{\circ} \mathrm{C}$ in buffer containing $50 \mathrm{mM}$ Tris- $\mathrm{HCl}, 50 \mathrm{mM} \mathrm{KCl}, 1 \mathrm{mM}$ ( $\mathrm{Na}_{2}$ EDTA), $1 \mathrm{mM}$ DTT, 9\% glycerol, $5 \mathrm{mM} \mathrm{MgCl}_{2}, \mathrm{pH}=$ 7.5. The reaction mixture contained $10 \mathrm{nM}$ WT APE1 or SNP forms, $1 \mu \mathrm{M}$ F-containing DNA duplex, and $1 \mu \mathrm{M}$ effector protein (OGG1, UNG, AAG, Pol $\beta$, XRCC1 or PCNA).

\section{Results and Discussion}

The analysis of the effect of different BER proteins on the activity of WT APE1 and SNP variants revealed several differences in protein-protein interactions. It was shown that OGG1, AAG, Pol $\beta$ and XRCC1 stimulate WT, R237A and P311S APE1, but revealed negligible effect on other SNP variants (R221C, N222H, G241R, M270T, R274Q), indicating that these amino acids can be involved in the protein-protein interactions during damage repair. Interestingly to note that UNG and PCNA slightly stimulate WT APE1, but at the same time decrease the activity of R221C, M270T and R274Q, and have no effect on N222H and G241R. Previously it was shown that APE1 G241R has 
little activity on nucleosomal DNA in comparison to WT APE1. Obtained data support that enzymes involved in the BER pathway have mutual effect on the activity of each other and that some amino acid substitution caused by SNPS lead to change of this effect.

TABLE 1 - AMINO ACID SUBSTITUTIONS ANALYZED IN THIS WORK

\begin{tabular}{|c|c|}
\hline $\begin{array}{l}\text { Amino acid } \\
\text { substitution }\end{array}$ & Location \\
\hline $\mathrm{R} 221 \mathrm{C}$ & $\begin{array}{c}\text { DNA-binding site (distance } 8.0 \AA \text { between } \mathrm{N} \eta \mathrm{l} \text { of } \mathrm{Arg} 221 \text { and } \mathrm{O} \text { atom of } 3^{\prime} \text {-phosphate group of second } \\
\text { nucleotide on } 3^{\prime} \text { side of F-site). }\end{array}$ \\
\hline $\mathrm{N} 222 \mathrm{H}$ & $\begin{array}{l}\text { DNA-binding site (distances } 4.5 \text { and } 5.0 \AA \text { between } \mathrm{N} \delta 2 \text { atom of Ans } 222 \text { and two } \mathrm{O} \text { atoms of } 5^{\prime}-\text { and }^{\prime}{ }^{\prime}- \\
\text { phosphate groups of second nucleotide on } 3^{\prime} \quad \text { side of F-site). }\end{array}$ \\
\hline R237A & 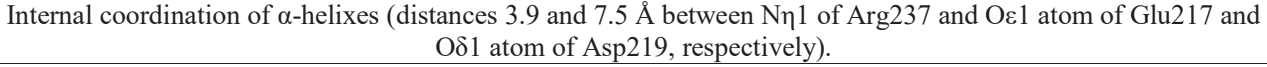 \\
\hline G241R & Exterior of protein globule. \\
\hline M270T & Active site. Met 270 is embedded into DNA minor groove, thereby displacing base opposite to AP site. \\
\hline R274Q & $\begin{array}{l}\text { Internal coordination near active site (distances } 2.6 \text { and } 3.0 \AA \text { between } \mathrm{N \eta} 1 / \mathrm{N \eta} 2 \text { of Arg2 } 24 \text { and carbonyl O } \\
\text { atoms of Ala304 and Ser307, respectively). }\end{array}$ \\
\hline P311S & Structural element at end of $\beta$-sheet, bordering catalytic loop. \\
\hline
\end{tabular}

\section{ACKNOWLEDGMENT}

This work was supported by a grant MD-3775.2019.4. 